\title{
RECENT RESULTS IN TOPOLOGICAL GRAPH THEORY*
}

\author{
By \\ F. HARARY (Ann Arbor, USA) \\ (Presented by A. RÉNYI)
}

A graph $G$ is usually defined as a finite collection $V$ of points together with a collection $X$ of lines, each of which joins two distinct points and no two of which join the same pair of points. This combinatorial definition asserts nothing about drawing graphs on surfaces such as the plane, sphere, torus, projective plane etc. The purpose of this lecture is to explore some of these topological aspects of graph theory and to describe a few unsolved problems concerning them.

In order to fix the terminology of this lecture, we begin by drawing all the graphs with four points:
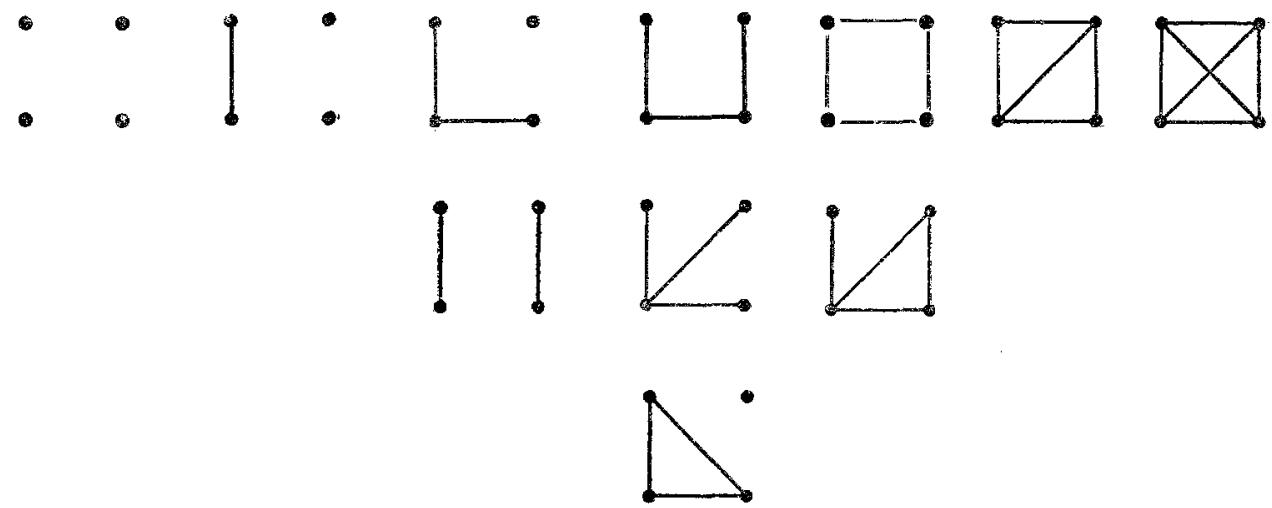

Fig. 1

Reading from left to right and top to bottom, the first of these graphs is called totally disconnected: it has four points and no lines. The last is the complete graph $K_{4}$ with four points; every pair of its points are adjacent. There are eleven different (non-isomorphic) graphs with four points, six of which are connected. The first two of these graphs having three lines are trees. The first of the two graphs with four lines is a cycle. We note that in none of these graphs does there occur any loops or parallel lines as shown in Figure 2.

loop

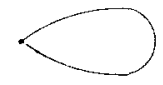

parallel lines

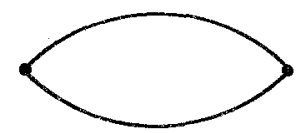

Fig. 2

* Lecture delivered before the János Bolyai Mathematical Society in Budapest on June 26, 1963. The preparation of this article was supported in part by the National Science Foundation, U. S. A. under grant NSF GP-207. 
A planar graph is one which can be drawn in the plane in such a way that no two of its edges intersect, a plane graph is already so drawn. In Figure 3 the complete graph $K_{4}$ is redrawn as a plane graph differently than in Figure 1, so that it is obviously planar.

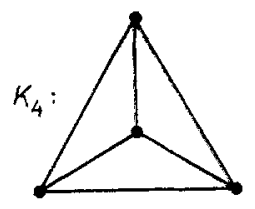

Fig. 3

The complete bicolored graph $K_{m, n}$ consists of $m$ points of one color, say light, and $n$ points of another color, say dark, in which two points are adjacent if and only if they have different colors. In Figure 4 , both the complete graph $K_{5}$ and the complete bicolored graph $K_{3,3}$ are shown. It is easy to verify that neither of these graphs is planar.
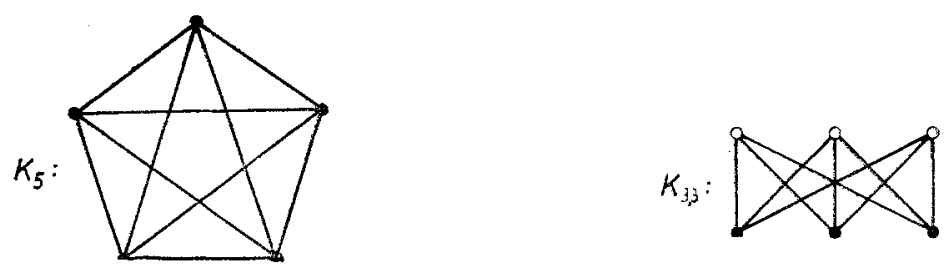

Fig. 4

Two graphs are isomorphic if there is a $1-1$ correspondence between their sets of points which preserves adjacency. The degree of a point is the number of lines with which it is incident. Two graphs are homeomorphic if it is possible to insert new points of degree 2 into their lines in such a way that the two resulting graphs are isomorphic. A graph homeomorphic with $K_{4}$ is shown in Figure 5.

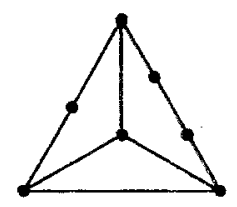

Fig. 5

With the help of these definitions we may state the first theorem of topological graph theory, due to Kuratowskr [13].

THEOREM 0. A graph $G$ is planar if and only if it has no subgraph homeomorphic with $K_{5}$ or $K_{3,3}$. 
We illustrate Theorem 0 in Figure 6(a) with the graph known as the Peterson graph. In Figure 6(b), we see that this graph is not planar, since it contains a subgraph homeomorphic with $K_{3.3}$ as shown by the light and dark points. Surprisingly, this graph which superficially resembles $K_{5}$ in appearance does not contain any subgraph homeomorphic with $K_{5}$.

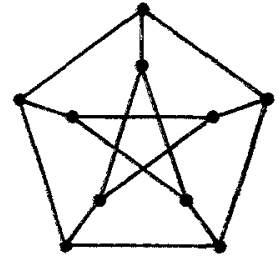

a)

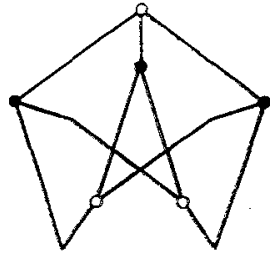

b)

Fig. 6

If a graph with a given number $p$ of points has a sufficiently large number of lines, then it is not planar. The next little theorem, described in HARARY [9], states this precisely.

THEOREM 1. If a graph $G$ has p points, $q$ lines and $q>3 p-6$, then $G$ is not planar.

The proof uses Euler's polyhedron formula $V-E+F=2$. In a triangulated plane graph each edge is on two faces and each face has three edges, so that $3 F=$ $=2 E$. Hence in such a graph $q=3 p-6$.

Corollary 1a. $K_{5}$ is not planat.

This is verified at once, since $K_{5}$ has $q=10$ and $3 p-6=9$.

The thickness $t(G)$ of a graph $G$ (a term introduced and studied by TUTTE [16]) with at least one line is the minimum number of planar subgraphs whose union is $G$. For example $t\left(K_{5}\right)=2$ and $t\left(K_{3,3}\right)=2$.

CoRollary $1 \mathrm{~b}$. For any graph $G$ with $p$ points and $q$ lines,

$$
q \leqq(3 p-6) t(G) \text {. }
$$

Of course every planar graph with at least one line has thickness 1 . It is easy to verify that $t\left(K_{8}\right)=2$ by drawing a planar graph $G$ with 8 points whose complement $G$ is also planar. Only recently it has been shown by exhaustion that $t\left(K_{9}\right)=3$; see Battle, Harary and Kodama [2], and TutTe [15]. The following table, which will be called Figure 7, lists all the known thicknesses of complete graphs, $K_{p}$, for $p=2$ to 33 .

\begin{tabular}{|c|c|c|c|c|c|c|c|c|c|}
\hline$p$ & $2-4$ & $5-8$ & $9-15$ & 16 & $17-21$ & 22 & $23-28$ & $29-33$ & 34 \\
\hline$t\left(K_{p}\right)$ & 1 & 2 & 3 & $?$ & 4 & $?$ & 5 & 6 & $?$ \\
\hline
\end{tabular}

Fig. 7 
The entries for $n=13,14$, and 15 cost me one shilling each, since I wagered L. W. BeINEKE that these thicknesses would be 4 and he was able to construct a decomposition of each of these three complete graphs as the union of three planar subgraphs. Of course, knowing in advance that $t\left(K_{9}\right)=3$ and $t\left(K_{15}\right)=3$, it would not be necessary to demonstrate that this is the value for $p=13$ and 14 . The smallest complete graph whose thickness is not yet known is $K_{16}$. I would conjecture that $t\left(K_{16}\right)=4$, and that it would require an argument similar to that which settled the thickness of $K_{9}$, but even more exhausting because of the considerably increased number of points of the graph. In the next theorem, BeINEKE and HARARY [4], the thickness of five-sixths of all the complete graphs is determined exactly.

THEOREM 2. The thickness of the complete graph $K_{p}$ is given by

$$
t\left(K_{p}\right)=\left[\frac{p+7}{6}\right] \text { for } p \equiv 0,1,2,3,5(\bmod 6), p \neq q .
$$

The entries in Figure 7 starting with $n=17$ were first obtained from this theorem.

A graph is bicolorable if its points can be colored, using two colors, in such a way that only two points having different colors are adjacent. It is well known that a graph is bicolorable if and only if it contains no cycles of odd length. Therefore, in particular, such a graph contains no triangles and satisfies the hypothesis of the next little theorem, whose proof is entirely analogous with that of Theorem 1 .

THEOREM 3. If $G$ has no triangles and $q>2 p-4$, then $G$ is not planar.

Corollary 3a. $K_{3,3}$ is not planar.

CoROllaRY $3 b$. If $G$ has no triangles, then

$$
q \leqq(2 p-4) t(G)
$$

The inequalities (1) and (3) and the concept of thickness are generalized in [5].

Applying the inequality (3) to the complete bicolored graph, which is of course bicolorable, we obtain the next inequality. Let $\{x\}$ be the smallest integer not less than $x$, and as usual let $[x]$ be the largest integer not exceeding $x$.

COROLlary 3c. A lower bound for the thickness of the complete bicolored graph is given by

$$
t\left(K_{m, n}\right) \geqq\left\{\frac{m n}{2(m+n-2)}\right\} .
$$

As reported in BEINEKE, HARARY and MOON [7], one can construct a family of graphs to show that most of the time the equality in the preceding corollary holds.

THEOREM 4. The thickness of the complete bicolored graph is given by

$$
t\left(K_{m, n}\right)=\left\{\frac{m n}{2(m+n-2)}\right\}
$$


except possibly when $m, n$ are both odd and there exists a positive integer $k$ such that

$$
n=\left[\frac{2 k(m-2)}{m-2 k}\right] \text {. }
$$

The smallest complete bicolored graph whose thickness is not yet known is $K_{17,21}$.

An orientable surface may be regarded as a sphere with handles on it (or holes in it); its genus is the number of handles. The genus $\gamma(G)$ of a graph $G$ is defined as the minimum genus of an orientable surface on which $G$ can be drawn with no two of its edges intersecting. Thus Theorem 0 says that a graph has genus 0 if and only if it contains no subgraph homeomorphic with $K_{5}$ or $K_{3,3}$. In Figure 8, we see that $\gamma\left(K_{5}\right)=\gamma\left(K_{3,3}\right)=1$, i. e. that these two graphs are foroidal. In this figure a torus is represented in the usual way as a rectangle in which both pairs of opposite sides are identified with each other as indicated by arrows.
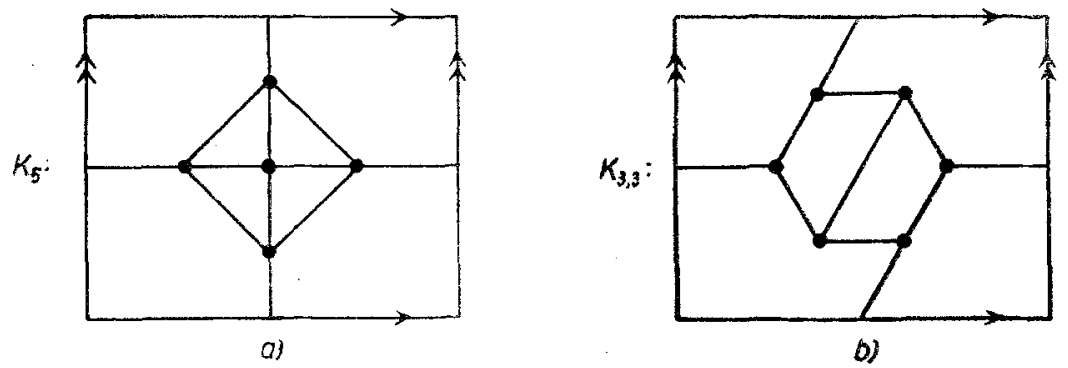

Fig. 8

Nobody knows the generalization of Theorem 0 to graphs of positive genus, not even for toroidal graphs. The question is: $G$ is toroidal if and only if $G$ does not contain homeomorphs of which subgraphs? It is not even known whether the family of such exceptional graphs is finite.

In order to embed graphs (without intersecting edges) in surfaces of large genus, COHEN, HARARY and KoDAMA [8] have developed a new method of representing an orientable surface by identifying appropriate edges of a pair of oriented polygons. They then illustrate by embedding $K_{7}$ in $S_{1}$ (a sphere with one handle), $K_{8}$ in $S_{2}$, and $K_{9}$ in $S_{3}$. An especially interesting problem involved the determination of $\gamma\left(K_{n}\right)$. In a sense, this problem is one-half solved.*

THEOREM 5. The genus of a complete graph is

when $n \equiv 0,3,4,5,7,10(\bmod 12)$.

$$
\gamma\left(\boldsymbol{K}_{n}\right)=\left\{\frac{(n-3)(n-4)}{12}\right\}
$$

* J. W. T. Youngs has just informed me that he has also proved equation (5) when $n=12 s+1$, so that equation (5) is now $7 / 12$ verifed. 
The proof of this result for $n \equiv 3,5,7,10(\bmod 12)$ appears in the work of RINGEL [14]. For $n \equiv 0(\bmod 12)$, the formula $(5)$ was demonstrated by J. W. T. Youngs (unpublished) and its validity for $n \equiv 4(\bmod 12)$ was recently established by Gustin (unpublished).

The next result, also found by Youngs et al [1], [17], is interesting because it relates the genus of a graph with the question of embedding it in a nonorientable surface. The theorem was proved by constructing an appropriate family of graphs $G_{n}$.

THEOREM 6. For any positive integer $n$, there exists a graph $G_{n}$ of genus $n$ which can be embedded in the projective plane.

A cut point of a connected graph is one whose removal results in a disconnected graph. A block of a graph $G$ is a maximal connected subgraph of $G$ containing no cut points of itself. The next result, proved only after extensive collaborative efforts, expresses the genus of a graph in terms of the genuses of its blocks; see BATTLE, Harary, Kodama and Youngs [3].

THEOREM 7. The genus of any graph is the sum of the genuses of its blocks.

A graph is $n$-connected if it is not disconnected by the removal of any $n-1$ of its points. Thus a block of a graph is a maximal 2-connected subgraph if it has more than two points. Recently, the preceding theorem has been generalized slightly by HARARY and KoDAMA [12] to the case in which a given graph is the union of exactly two maximal $n$-connected subgraphs. Further generalization appears to be a very subtle problem.

The connectivity of a graph $G$ is $n$ if $G$ is $n$-connected but not $(n+1)$-connected. We have recently [10] obtained a formula for the greatest connectivity among all graphs with a given number of points and lines.

THEOREM 8. The maximum connectivity among all graphs with $p$ points and $q$ lines, is $\left[\frac{2 q}{p}\right]$, provided $q \geqq p-1$.

For a given graph $G$, its crossing number $c(G)$ is the minimum number of pairwise intersections of its edges when $G$ is drawn in the plane. The crossing number of the complete bicolored graph was determined exactly by ZaRANKIEWICZ [18]:

$$
\left\{\begin{array}{l}
c\left(K_{2 m, 2 n}\right)=\left(m^{2}-m\right)\left(n^{2}-n\right) \\
c\left(K_{2 m, 2 n+1}\right)=\left(m^{2}-m\right) n^{2} \\
c\left(K_{2 m+1,2 n+1}\right)=m^{2} n^{2}
\end{array}\right.
$$

However, the crossing number of the complete graph remains an open problem. It can be shown by an explicit construction that an upper bound for $c\left(K_{n}\right)$ is given by

$$
c\left(K_{n}\right) \leqq\left\{\begin{array}{lll}
\frac{1}{64}(n-1)^{2}(n-3)^{2} & n & \text { odd } \\
\frac{1}{64} n(n-4)(n-2)^{2} & n & \text { even }
\end{array}\right.
$$


as mentioned in HARARY and HILL [11]. The concensus of opinion is that the conjecture, which asserts that this upper bound is the exact value of $c\left(K_{n}\right)$, is correct. But it has not even been proved that $c\left(K_{n}\right)$ approaches $n^{4} / 64$ for large $n$.

Let $Q_{n}$ be the (graph of the) $n$-cube, so that $Q_{n}$ has $2^{n}$ points each being a binary sequence $a_{1} a_{2} \ldots a_{n}$ (of zeros and ones). Two points of $Q_{n}$ are adjacent whenever their sequences differ in exactly one place. We have recently obtained [6] an exact formula for its genus.

THEOREM 9. The genus of the n-cube is

$$
\gamma\left(Q_{n}\right)=(n-4) 2^{n-3}+1 .
$$

Added in proof. A shrinking of a graph is the result of replacing a line by a single point. A contraction of a graph is obtained from a sequence of shrinkings. The following criterion for planarity [19] may be readily derived from Kuratowski's theorem.

Theorem 10. A graph is nonplanar if and only if it has $K_{5}$ or $K_{3,3}$ as a subgraph of a contraction.

For example it is immediately apparent that the PETERson graph shown in Figure $6\left(\right.$ a) has $K_{5}$ as a contraction and hence is nonplanar.

THE UNIVERSTTY OF MICHIGAN

AND UNTERSITY COLLEGE, LONDON

(Received 24 October 1963)

\section{References}

[1] L. Auslander, T. A. Brown and J.W. T. Youngs, The imbedding of graphs in manifolds, J. Math. Mech., 12 (1963), pp. 629-634.

[2] J. Battle, F. Harary and Y. KodAMA, Every planar graph with nine points has a nonplanar complement, Bull. Amer. Math. Soc., 68 (1962), pp. 569-571.

[3] J. Battle, F. Harary, Y. Kodama and J. W. T. Youngs, Additivity of the genus of a graph, Bull. Amer. Math. Soc., 68 (1962), pp. 565-568.

[4] L. W. Benneke and F. Harary, On the thickness of the complete graph, Bull. Amer. Math. Soc., 70 (1964), pp. 618-620. The thickness of the complete graph. To appear in Canadian J. Math.

[5] L. W. BEINEKE and F. HARARY, Some inequalities involving the genus of a graph and its thicknesses, to appear in Proc. Glasgow Math. Assoc., 1964.

[6] L. W. BEINEKE and F. HARARY, The genus of the $n$-cube, to appear in Canadian J. Math.

[7] L. W. Beineke, F. Harary and J. W. Moon, On the thickness of the complete bipartite graph, Proc. Camb. Phil. Soc., 60 (1964), pp, $1-5$.

[8] D. E. Cohen, F. Harary and Y. Kodama, On the embedding of complete graphs in orientable surfaces, Mathematika, 10 (1963), pp. 79-83.

[9] F. Harary, A complementary problem on nonplanar graphs, Math. Mag., 35 (1962), pp. $301-303$.

[10] F. Harary, The maximum connectivity of a graph, Proc. Natl. Acad. Sci., U. S. A. 48 (1962), pp. $1142-1146$.

[11] F. Harary and A. Hill, On the number of crossings in a complete graph, Proc. Edinburgh Math. Soc., 13 (1963), pp. 333-338.

[12] F. HARARY and Y. KODAMA, On the genus of an n-connected graph, Fund. Math., 54 (1963). pp. $7-13$ 
[13] C. Kuratowski, Sur le problème des courbes gauches en topologie, Fund. Math, 15 (1930), pp. $271-283$.

[14] G. RiNGEL, Färbungsprobleme auf Flächen und Graphen (VEB Deutscher Verlag der Wissenschaften, Berlin, 1959), Über das Problem der Nachbargebiete auf orientierbaren Flächen, Abhandlung Maih. Sem. Univ. Hamburg, 25 (1961), pp. 105-127.

[15] W. T. Tutre, The non-biplanar character of the complete 9-graph, Canad. Math. Bull, 6 (1963), pp. $319-330$.

[16] W. T. TUTTE, The thickness of a graph, Indag. Math., 25 (1963), pp. 567-577.

[17] J. W. T. YounGs, Minimal embeddings and the genus of a graph, J. Math. Mech., 12 (1463), pp. $303-315$.

[18] K. ZaRANKIEWICZ, On a problem of P. Turán concerning graphs, Fund. Math., 41 (1954), pp. $137-145$.

[19] F. Harary and W. T. TutTE, A dual form of Kuratowski's theorem, to appear in Canadian Math. Bill. 